\title{
3D-printed, home-made, UV-LED photoreactor as a simple and economic tool to perform photochemical reactions in high school laboratories
}

\author{
${ }^{1}$ Dipartimento di Chimica, Università degli Studi di Milano Via Colgi 19, 20133, Milano, Italy, E-mail: sergio.rossi@unimi.it. \\ https://orcid.org/0000-0002-2694-9535. \\ ${ }^{2}$ Istituto Statale di Istruzione Superiore "Paolo Carcano", Via Castelnuovo 5, 22100 Como, Italy
}

\begin{abstract}
:
In the paper we present the simple manufacturing of an easy and economical UV-A photoreactor using a desktop 3D printer and its application in chemical transformations. PLA (polylactic acid) was used as inexpensive and not toxic polymer for the 3D printing process, while commercially available decorative ultraviolet LEDs (UV-A) have been employed as a light source. With this device, the photoreduction of benzophenone was performed in high yield and short times, compatible with the duration of a typical laboratory experiment in a high school program.
\end{abstract}

Keywords: 3D printing, benzophenone, photoreactor, photoreduction, UV-LEDs

DOI: $10.1515 /$ cti-2019-0010

\section{Introduction}

In the last years, the scientific community have started to develop many environmentally friendly technologies through the use of "green" solutions which nowadays are important tools for continuous improvement in the quality of modern life. In order to bring young people closer to these technologies, educational institutions play an extremely important role in the diffusion of sustainable concepts, since they represent one of the first places to come into contact with these modern technologies. In this contest, the usage of natural resources such as the light could offer a lot of opportunities in the development of "green" solutions to modern problems.

Photochemistry represents the study of chemical reactions that occur under the influence of visible and /or ultraviolet light (Noyes \& Kassel, 1926; Oelgemoller, 2016; Ramamurthy \& Turro, 1993). If the absorption of light is sufficient to introduce enough energy in a molecule, the resulting excited electronic state may determine a reorganization of the molecule itself and new bond breaking/forming phenomena can be observed.

According to the Planck's law, the value of a quantum of energy (E) depends on the frequency $v$ of the radiation according to the equation $\mathrm{E}=\mathrm{h} v$ (where $\mathrm{h}$ is the Planck constant and $\nu$ is the frequency calculated as the ratio between the speed of light and the wavelength $\lambda$ ). Therefore, photons characterized by longer wavelengths (and relatively lower frequencies) are less energetic. As a consequence, ultraviolet radiation (wavelength from 200 to $400 \mathrm{~nm}$ ) is more energetic compared to visible light (from 400 to $800 \mathrm{~nm}$ ) and it can be considered the "light of choice" to study photochemical transformations. However, it is worth reminding that, of the solar radiation reaching the Earth, $5 \%$ is UV light, $50 \%$ is visible light and the remaining part is composed of infrared and longer wavelengths radiation.

These concepts are objects of chemical/physical studies in high schools, but often, despite their importance they remains only abstract descriptions of phenomena may sound very unfamiliar to most people. To overcome this fact, one of the most effective way of reinforcing new concepts is through hands-on experience. From this point of view, one classical photochemical experiment that can be reproduced in a high school is the photoreduction of benzophenone promoted by light. This process involves a reductive homo-coupling of an aromatic carbonyl compound to produce a symmetrically substituted 1,2-diol and represents an evolution of Demselben and Fittig observations (Demselben, 1859; Fittig, 1860) which demonstrated that aldehydes and ketones can be reduced to the corresponding homo-alcohols when treated with alkaline solutions (Pinacol Coupling reaction) 
(Figure 1). Fittig reported that, upon treatment of a carbonyl derivative with an alkaline earth metal (such as $\mathrm{Mg}$ ), the generated radical intermediate undergoes a coupling reaction via carbon-carbon bond formation to give the corresponding 1,2-diol. In the early 1900s Prof. G. Ciamician (an Italian chemist who worked at the University of Bologna and which is considered the "father of organic photochemistry") demonstrated that the single electron transfer process (SET) necessary for the activation of the carbonyl bond can be also performed by light without the presence of the metal. According to his studies, a methanol solution of benzophenone, when exposed for 8 days to sunlight can be converted into a solution of benzopinacol (1,1,2,2-tetraphenyl-1,2ethandiol) in $90 \%$ yield (Ciamician \& Silber, 1900).

\section{Pinacol coupling}

a Demselben and Fittig work

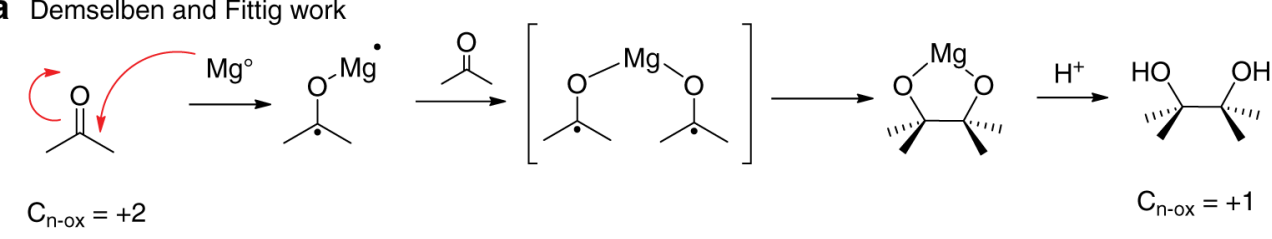

b Ciamician work

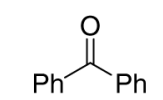

Benzophenone

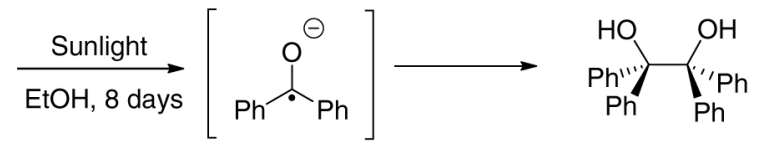

Benzopinacol $y=90 \%$

Figure 1: Homo-reductive coupling of ketones.

Starting from these pioneering studies, many other photochemical transformations have been developed in a very efficient and "green" way.

Unfortunately, even if sunlight is essentially free, it is not continuously available (no light during the night!) and reactions mediated by sunlight generally take days or weeks to be completed. In order to overcome this problem, many scientists have started to investigate different sources of lights and nowadays, the synthesis of benzopinacol can be performed in $92 \%$ yield upon UV-light irradiation of a solution of benzophenone in $2-$ propanol in $24 \mathrm{~h}$ only (2-propanol acts as both solvent and reducing agent) (Fieser, Fieser, \& Williamson, 1979; Pavia, Kriz, \& Lampman, 1976; Pavia, Kriz, Lampman, \& Engel, 2012). Interestingly, the yield of the photoreductive process is comparable to that observed by Ciamician using sunlight, even if the reaction can be carried out in a much shorter time.

With the aim to perform the photoreduction of benzophenone into educational laboratories in a high school, and thanks to the availability of an economic 3D printer in the school, we realized a low-cost, home-made, 3D-printed UV-LEDs photoreactor apparatus. This device costed approximately 20 euros and can be safely used by students in a standard chemistry laboratory. As a light source, commercially available and inexpensive ultraviolet LEDs (385-400 nm, UV-A level) employed for decorative lighting were used.

\section{Realization of the photoreactor device}

3D printing (or additive manufacturing) is any of various processes used to make a real three-dimensional object from a virtual design generated by a Computer-Aided Design (CAD) software. This enabling technology has found extensive applications in several fields including organic synthesis (Rossi, Puglisi, \& Benaglia, 2018) but it started to be recognized as important tool for teaching purposes, since it offers an opportunity to visualize chemical structures and properties through the realization of "ad-hoc" devices (Pinger, Castiaux, Speed, \& Spence, 2018; Tabassum et al., 2018) and tangible models of molecules, orbitals and surfaces (de Cataldo, Griffith, \& Fogarty, 2018; Robertson \& Jorgensen, 2015; Rossi, Benaglia, Brenna, Porta, \& Orlandi, 2015). Following our interest in the application of 3D printing for teaching purposes (Rossi et al., 2015), we herein report the realization of an inexpensive 3D-printed photoreactor by a cheap Sharebot NG desktop 3D printer (website: https:/ / www.sharebot.it/stampanti-3dsharebot-next-generation/).

The initial structure of the photoreactor was designed using Autodesk 123D Design software, (123d Design, Version 2.2.14. Downloaded from www.autodesk.com on March 2017, a free licensed software which allows to easily perform combine, subtract and intersect operations between solids, according to the WYSIWYG approach (What You See Is What You Get). The photoreactor was designed with an external diameter of $13 \mathrm{~cm}$, in order 
to fit with the most common magnetic stirrer plates thus ensuring the stirring of liquid samples during the irradiation process; it is $2 \mathrm{~cm}$ height and presents an internal diameter of $12.6 \mathrm{~cm}$, able to host a short strip of LEDs. In addition, the central ring nut is able to host $7+1$ vials (the central position is black to light in order to perform control experiments) with an approximately $2.5 \mathrm{~cm}$ vial/led distance, in order to ensure high reproducibility. The virtual design of the photoreactor structure (Figure 2a) was then subjected to a slicing process performed using free Slic3r 1.4 software (website: https://slic3r.org/). The new file generated (.gcode) containing all the information required for the printing process was finally sent to the 3D printer (see supporting information for further details). The photoreactor was printed in $2.15 \mathrm{~h}$ using the desktop 3D printer; the results are showed in Figure $2 \mathrm{~b}$. For the 3D printing process, a $1.75 \mathrm{~mm}$ PLA (PolyLactic Acid) thermopolymer filament was selected since it is less expensive compared to other materials and presents a good safety profile (the material and the fumes generated during the heating process present low/no toxicity). Notably, no supports are required during the printing process and, as results, printing waste are avoided. An adhesive reflective strip sheet was applied internally to the photoreactor in order to avoid light radiation dispersion.
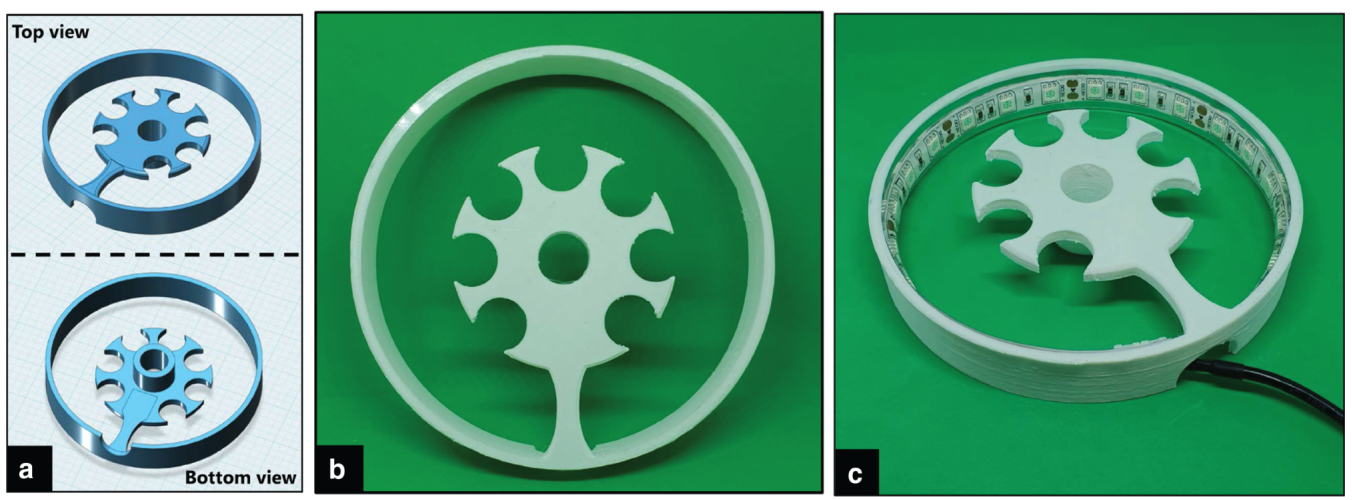

Figure 2: (a) Computer design of the photoreactor realized with Autodesk 123D Design software; (b) 3D-printed photoreactor; (c) home-made 3D-printed UV-LEDs photoreactor.

The choice of the light source was crucial. Many sources of ultraviolet light are available in the market (UV lamps, "sun" lamps, "black lights", fluorescent bulbs, etc.) but they present some disadvantages such as the mandatory presence of cooling systems and their high costs, often prohibitive for an undergraduate laboratory tight budget. To overcome these problems, we focused our attention to commercially available IP95 waterproof ultraviolet Light Emitting Diodes, commonly named LEDs (385-400 nm, UV-A level) employed for decorative lighting, which are available at very low price. Generally, LEDs do not require high voltage (DC 12V or 24V), they are touchable and durable (approximately 50,000 h life, with high power efficiency) and present excellent heat dissipation. Indeed, thanks to their low wattage, LEDs can be turned on and off without warm-up/cooldown cycle times that are usually required for higher wattage systems.

We selected on the market a "plug and play system" without extra wiring connection, with a self-adhesive tape that holds the strip light safely and securely to the photoreactor support (we preferred LED strips that could be cut). The LEDs wavelength emission profile together with their specific light intensity (expressed as $\mathrm{mWcm}^{-2}$ ) have been determined before their use. In particular, the emission spectrum of the investigated UVA model has been compared with those of two other LEDs models, i.e. mainly emitting at relatively larger wavelengths. In particular, all the spectra have been obtained by using a compact CCD spectrometer (model CCS200/M) connected to a multimode optical fiber, purchased from Thorlabs. As clearly depicted in Figure 3a, each investigated type of LEDs is characterized by an almost monochromatic emission profile (with a full width at half-maximum intensity of ca. 10-20 nm) showing a maximum of intensity located at ca. 390, 450 and $510 \mathrm{~nm}$ for the UV-A, the Blue- and the Green- LEDs model, respectively. The light power intensity was thus checked using a Thorlabs PM200 power meter equipped with a S130VC power head with a Si detector. The measured light intensities (see Figure 3b), though slightly decreasing by moving the maximum of LEDs emission towards longer wavelenghts, resulted to be quite weak, thus preventing any undesired photobiological risks according to IEC/TR 62471-2:2009 normative (International Electrotechnical Commission (IEC), technical report "Photobiological safety of lamps and lamp systems part 2", website: https://webstore.iec.ch/publication/7074). 
a

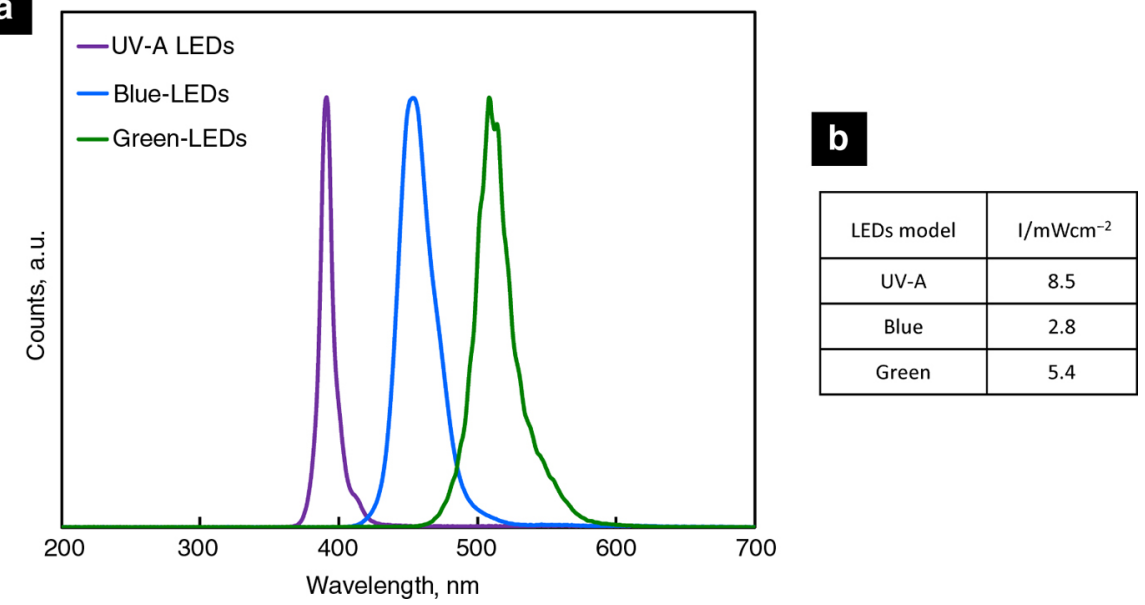

Figure 3: Comparison of (a) the normalized emission profiles and (b) the measured intensities of the investigated UVA-, Blue- and Green-LEDs.

A strip of 21 leds $(35 \mathrm{~cm})$ was then stuck to the photoreactor and used to investigate the photoreduction of benzophenone. The assembled photoreactor is showed in Figure 2c. Costs and the detailed assembly procedure of the photoreactor are reported in the supplementary information.

\section{Experimental methods and results}

This low-cost photochemical equipment has been successfully employed by high school students in the investigation of benzophenone photoreduction reaction promoted by LEDs light. It is known that $\mathrm{SiO}_{2}$ glass labware absorbs light with $\lambda<210 \mathrm{~nm}$, however since these experiments were conducted at higher wavelengths, no interference was observed when standard disposal glass vials were used as reaction vessels. In a typical experiment, a solution of benzophenone in $2 \mathrm{~mL}$ of 2-propanol was subjected to LEDs irradiation for a period 3-24 $\mathrm{h}$ (see Table 1). Depending on the concentration of the solution and the wavelength of light, the formation of benzopinacol (1,1,2,2-Tetraphenyl-1,2-ethanediol) was observed as a suspension of a white crystalline powder by naked eyes, which remains almost undissolved in 2-propanol (Figure 4).
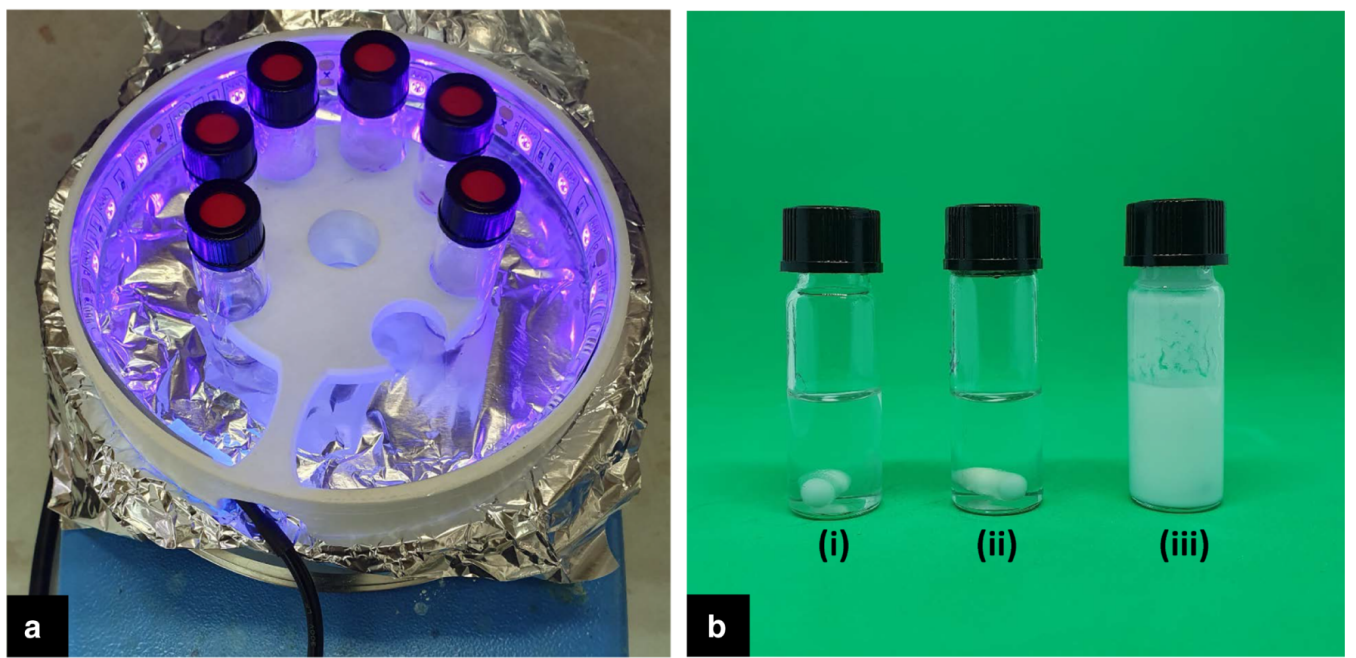

Figure 4: (a) Typical setup for the irradiation of benzophenone solutions by UV-LEDs. An aluminium foil is added in order to increase light reflection (b) comparison between a benzophenone solution in a vial equipped with magnetic bar irradiated for $24 \mathrm{~h}$ with (i) green LEDs (ii) blue LEDs and (iii) UV-A LEDs.

Reactions were also monitored by TLC analysis on silica plates (eluent 8:2 hexanes:ethyl acetate) dissolving a small amount of the crude reaction in acetonitrile prior deposition. Results are collected in Table 1.

Table 1: Results obtained in the photoreduction of benzophenone performed using 3D printed photoreactor. 


\begin{tabular}{|c|c|c|c|c|}
\hline $\begin{array}{l}\text { Benzophenone } \\
{[\mathrm{M}]}\end{array}$ & Light source & $\begin{array}{l}\text { Time required to observe } \\
\text { precipitation under LED } \\
\text { irradiation, } h\end{array}$ & Yield after $3 \mathrm{~h}, \%^{\mathrm{a}}$ & $\begin{array}{l}\text { Yield after } 24 \\
\text { h, } \%{ }^{a}\end{array}$ \\
\hline \multirow[t]{3}{*}{0.5} & UV-A LEDs & 2.00 & 12 & 81 \\
\hline & Blue-LEDs & $\begin{array}{l}\text { No precipitation observed } \\
\text { after } 24 \mathrm{~h}\end{array}$ & - & - \\
\hline & Green-LEDs & $\begin{array}{l}\text { No precipitation observed } \\
\text { after } 24 \mathrm{~h}\end{array}$ & - & - \\
\hline \multirow[t]{3}{*}{0.4} & UV-A LEDs & 2.00 & 12 & 76 \\
\hline & Blue-LEDs & $\begin{array}{l}\text { No precipitation observed } \\
\text { after } 24 \mathrm{~h}\end{array}$ & - & - \\
\hline & Green-LEDs & $\begin{array}{l}\text { No precipitation observed } \\
\text { after } 24 \mathrm{~h}\end{array}$ & - & - \\
\hline \multirow[t]{3}{*}{0.3} & UV-A LEDs & 2.10 & 16 & 75 \\
\hline & Blue-LEDs & $\begin{array}{l}\text { No precipitation observed } \\
\text { after } 24 \mathrm{~h}\end{array}$ & - & - \\
\hline & Green-LEDs & $\begin{array}{l}\text { No precipitation observed } \\
\text { after } 24 \mathrm{~h}\end{array}$ & - & - \\
\hline \multirow[t]{3}{*}{0.2} & UV-A LEDs & 2.30 & 16 & 72 \\
\hline & Blue-LEDs & $\begin{array}{l}\text { No precipitation observed } \\
\text { after } 24 \mathrm{~h}\end{array}$ & - & - \\
\hline & Green-LEDs & $\begin{array}{l}\text { No precipitation observed } \\
\text { after } 24 \mathrm{~h}\end{array}$ & - & - \\
\hline \multirow[t]{3}{*}{0.1} & UV-A LEDs & 3.15 & Traces & 34 \\
\hline & Blue-LEDs & $\begin{array}{l}\text { No precipitation observed } \\
\text { after } 24 \mathrm{~h}\end{array}$ & - & - \\
\hline & Green-LEDs & $\begin{array}{l}\text { No precipitation observed } \\
\text { after } 24 \mathrm{~h}\end{array}$ & - & - \\
\hline \multirow[t]{3}{*}{0.05} & UV-A LEDs & $\begin{array}{l}\text { No precipitation observed } \\
\text { after } 24 \mathrm{~h}\end{array}$ & Traces $^{b}$ & Traces $^{b}$ \\
\hline & Blue-LEDs & $\begin{array}{l}\text { No precipitation observed } \\
\text { after } 24 \mathrm{~h}\end{array}$ & - & - \\
\hline & Green-LEDs & $\begin{array}{l}\text { No precipitation observed } \\
\text { after } 24 \mathrm{~h}\end{array}$ & - & - \\
\hline
\end{tabular}

a Yield was determined by filtration and isolation of the crude powder. ${ }^{\mathrm{b}} \mathrm{TLC}$ analysis of the crude showed the formation of the desired product in traces amount.

No product formation was observed after $24 \mathrm{~h}$ using Blue or Green LEDs. A TLC analysis confirmed that only starting material was present in the solution. Gratefully, all the experiments performed using UV-A LEDs as a light source led to the formation of the desired product in modest yield even after $3 \mathrm{~h}$ only. The formation of the desired product was confirmed by the presence of a white precipitate observed in the reaction vessel. Benzopinacol was obtained in $81 \%$ yield when a $0.5 \mathrm{~m}$ solution of benzophenone in 2-propanol was irradiated for $24 \mathrm{~h}$. Even if, in our case, yields are not quantitative, it must be noted that no dry solvents or inert atmosphere were necessary to perform these experiments.

\section{Conclusions}

In conclusion, a low cost, home-made, 3D-Printed UV-LED photoreactor was realized and used to perform photochemical reactions in a high school laboratory. This device was used to successfully perform the reductive homo-coupling of benzophenone to benzopinacol, leading to the formation of the desired product up to $81 \%$ yield. The realization of this simple device was performed taking advantage of 3D printing technology combined with commercially available UV-A LEDs. We believed that this low-cost approach could facilitate the inclusion of photochemistry experiments in high schools laboratories with small budgets. 


\section{Supplementary Information}

Supplementary material consists in the .STL file containing the virtual design of the photoreactor and a document containing the $3 \mathrm{D}$ printing parameters used in the $3 \mathrm{D}$ printing process, as well as the typical procedure and setup followed to perform the homo-coupling of benzophenone described in the text.

\section{Acknowledgments}

The authors thanks Istituto Statale di Istruzione Superiore "Paolo Carcano", for providing laboratories and equipment. The authors thanks all the teachers of the Chemistry Department of the "Paolo Carcano" Institute attending to the "Nuove tecnologie per lo sviluppo di processi di sintesi innovativi e sostenibili" project.

Financial Support: S.R and A.P. thanks ITN-EID project Marie Sklodowska-Curie Actions Innovative Training Network - TECHNOTRAIN H2020-MSCA-ITN-2018 Grant Agreement n. 812944 for financial support. All the authors thanks PLS-2018 program of Università degli Studi di Milano.

\section{References}

Ciamician, G., \& Silber, P. (1900). Chemische Lichtwirkungen. Berichte der deutschen chemischen Gesellschaft, 33(3), 2911-2913. doi:10.1002/cber.19000330326.

de Cataldo, R., Griffith, K. M., \& Fogarty, K. H. (2018). Hands-on hybridization: 3D-printed models of hybrid orbitals. Journal of Chemical Education, 95(9), 1601-1606. doi:10.1021/acs.jchemed.8boo078.

Demselben. (1859). Ueber einige Metamorphosen des Acetons der Essigsäure. Annalen der Chemie und Pharmacie, 110(1), 23-45. doi:10.1002/jlac.18591100104.

Fieser, L. F., Fieser, L. F., \& Williamson, K. L. (1979). Organic experiments. Lexington, USA: D. C. Heath.

Fittig, R. (1860). 41. Ueber einige Derivate des Acetons. Annalen der Chemie und Pharmacie, 114(1), 54-63. doi:10.1002/jlac.18601140107.

Noyes, W. A., \& Kassel, L. S. (1926). A review of photochemistry. Chemical Reviews, 3(2), 199-225. doi:10.1021/cr60010a003.

Oelgemoller, M. (2016). Solar photochemical synthesis: From the beginnings of organic photochemistry to the solar manufacturing of commodity chemicals. Chemical Reviews, 116(17), 9664-9682. doi:10.1021/acs.chemrev.5bo0720.

Pavia, D. L., Kriz, G. S., Jr., \& Lampman, G. M. (1976). Introduction to organic laboratory techniques: A contemporary approach. Philadelphia: Saunders.

Pavia, D. L., Kriz, G. S., Lampman, G. M., \& Engel, R. G. (2012). A microscale approach to organic laboratory techniques. Boston, USA: Cengage Learning.

Pinger, C. W., Castiaux, A., Speed, S., \& Spence, D. M. (2018). Plate reader compatible 3D-printed device for teaching equilibrium dialysis binding assays. Journal of Chemical Education, 95(9), 1662-1667. doi:10.1021/acs.jchemed.8boo215.

Ramamurthy, V., \& Turro, N. J. (1993). Photochemistry: Introduction. Chemical Reviews, 93(2), 585-586. doi:10.1021/crooo18a600.

Robertson, M. J., \& Jorgensen, W. L. (2015). Illustrating concepts in physical organic chemistry with 3D printed orbitals. Journal of Chemical Education, 92(12), 2113-2116. doi:10.1021/acs.jchemed.5boo682.

Rossi, S., Benaglia, M., Brenna, D., Porta, R., \& Orlandi, M. (2015). Three dimensional (3D) printing: A straightforward, user-friendly protocol to convert virtual chemical models to real-life objects. Journal of Chemical Education, 92(8), 1398-1401. doi:10.1021/acs.jchemed.5boo168.

Rossi, S., Puglisi, A., \& Benaglia, M. (2018). Additive manufacturing technologies: 3D printing in organic synthesis. ChemCatChem, 10(7), 15121525. doi:10.1002/cctc.201701619.

Tabassum, T., Iloska, M., Scuereb, D., Taira, N., Jin, C., Zaitsev, V., \& Kim, T. (2018). Development and application of 3D printed mesoreactors in chemical engineering education. Journal of Chemical Education, 95(5), 783-790. doi:10.1021/acs.jchemed.7boo663.

Supplementary Material: The online version of this article offers supplementary material (DOI: https://doi.org/10.1515/cti-2019-0010). 